\title{
Campaña global para la participación y concienciación en materia de seguridad y salud
}

\section{Global campaign for participation and raising awareness in health and safety}

\section{Marta González del Río', Pedro Ledesma García'}

\section{FCC. España.}

\section{Correspondencia}

Pedro Ledesma García.

FCC. España

Correo electrónico: pfledesmag@fcc.es

Resumen

La campaña global se apoya en el sistema de gestión de FCC y la nueva reorganización del trabajo, que contribuye a mantener y reforzar el objetivo principal de "Accidentes 0". Con este objetivo y a partir de la nueva estructura y gestión, la compañía ha lanzado varias líneas de trabajo y ha realizado nuevas actuaciones dirigidas a fortalecer la integración de la cultura preventiva y mejorar los resultados. El compromiso y la unificación de procesos, la optimización de recursos, la comunicación y el estímulo de los empleados son los pilares fundamentales de esta iniciativa.

El objetivo de la campaña es fomentar la cultura preventiva a todos los niveles y favorecer la actitud positiva de los trabajadores hacia la prevención de riesgos laborales.

Palabras clave: Campaña global, Accidentes O, Cultura preventiva, Comunicación.

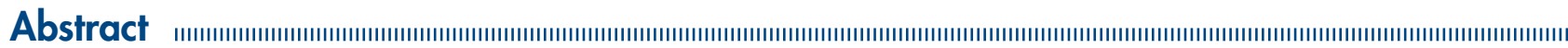

The global campaign is based on the FCC H\&S management system and the new reorganization work, that help to the maintenance and reinforcement of the main objective: " 0 Accidents". From the new structure and management, with this objective the company has launched several lines of work and has made new actions aimed to reinforce H\&S culture and to improve the results. The commitment and processes unification, adjusting resources, communication and employees incentive are the basic principles of this initiative.

The objective of this campaign is the encouraging of H\&S culture at all levels and the contributing to the positive attitude from workers to the H\&S issues.

Key words: Global campaign, O accidents, Prevention culture, Communication. 


\section{INTRODUCCIÓN}

FCC es una empresa especializada en servicios ciudadanos, con una producción altamente diversificada. Sus actividades básicas son la gestión de servicios medioambientales y agua, la construcción de grandes infraestructuras, la producción de cemento, equipamientos urbanos y la generación de energías renovables.

FCC está presente en más de 50 países, en los que da empleo a más de 70.000 personas. Entre sus actividades se destaca la recogida y tratamiento de 9 millones de toneladas de residuos domésticos al año; el préstamo de Servicios Ciudadanos en más de 5.000 municipios de todo el mundo; Aqualia, la división de agua, atiende a más de 28 millones de personas en 17 países; la construcción actualmente de más de 200 kilómetros de tren de Alta Velocidad; Cementos Portland tiene una capacidad de producción anual de 15 millones de toneladas de cemento; y se está gestionando, a través de Globalvía, 22 autopistas en Europa y América, con un total de $1.470 \mathrm{~km}$ de recorrido.

\section{PRL EN LA EMPRESA}

La mejora permanente de las condiciones de trabajo, la reducción de la accidentalidad y la promoción de la integración de la gestión preventiva ha sido siempre uno de los objetivos de la organización, para lo cual se cuenta con Sistemas de Gestión de prevención de riesgos laborales como herramienta básica de actuación, que aseguran que los empleados, sea cual sea su posición en la escala de mandos, puedan y deban participar en procesos clave como:

- La identificación de peligros y evaluación de riesgos.

- La investigación de incidentes.

- Los procesos de control y planes de inspección.

- Reuniones de control de la gestión y desarrollo y revisión de procesos como la planificación y la definición de objetivos.

Procesos que se destinan a asegurar el máximo nivel de seguridad y salud de los trabajadores, fomentando la integración de la prevención en los distintos procesos y responsabilidades de la organización.

Los objetivos de la empresa en materia de seguridad y salud son:

- Mejora permanente de las condiciones de trabajo.

- Reducción de la accidentalidad "Accidentes 0".

— Integración de la gestión Preventiva.

\section{PROYECTO}

\section{Antecedentes}

Distintos factores y la situación actual de crisis económica dificulta el continuar con el ritmo de mejora continua hasta ahora alcanzado, por ello surgen nuevas dificultades hacia la gestión de la seguridad y salud debido a las características cambiantes del trabajo, nuevos mercados, actividades y exigencias de la vida laboral; además del entorno generalizado de falta de optimismo para afrontar los nuevos retos y responsabilidades; el necesario control de recursos y gestión eficiente; y el afianzamiento del cambio y la concepción de la prevención como necesidad legal o burocrática, a la total integración de la misma dentro del resto de los procesos empresariales como una de las estrategias clave en la organización para su mayor eficiencia y mejora de resultados.

Se desvelan por tanto necesidades tales como afianzar el compromiso de la organización hacia la Seguridad y Salud; fomentar una cultura preventiva desde un liderazgo 
visible y activo de la Dirección, asegurando el compromiso a todos los niveles; la definición de líneas de trabajo comunes que contribuya a eliminar o minimizar los riesgos creados, permitiendo fijar objetivos de permanente mejora y de reducción de la accidentalidad; la selección y reorganización de los recursos, mejorando su eficacia y efectividad; fomentar las comunicaciones eficaces y la promoción de actitudes permitiendo a todos los trabajadores contribuir de una manera responsable en el ámbito de la seguridad y salud laboral; el fomento de la cultura preventiva en todos los entornos y hacia todos los grupos de interés; y premiar las buenas prácticas y estimular el esfuerzo por seguir mejorando.

\section{Desarrollo}

Las medidas implantadas para el compromiso y la unificación son la Política Corporativa de PRL para toda la compañía cuyos ejes fundamentales son la mejora permanente de las condiciones de trabajo y reducción de la accidentalidad, el compromiso de toda la organización, y la implicación de otros grupos de interés; el Manual Corporativo de Seguridad y Salud que describe los elementos y criterios básicos para la gestión de la prevención en todas las áreas y territorios, la definición de competencias y responsabilidades de todo el personal, y la actuación planificada y sistematizada que contribuya a eliminar o minimizar los riesgos creados por la actividad laboral; el Protocolo de Comunicación de Accidentes Graves cuyo objeto es mejorar, unificar y normalizar las pautas de actuación para la comunicación de accidentes graves, muy graves o mortales, a los distintos niveles de estructura de FCC y en todo su territorio de actuación; y el Código ético FCC donde se estipula el estricto cumplimiento de las normas de seguridad y salud para el grupo y sus colaboradores.

Para la optimización de recursos se lleva a cabo una reorganización de los recursos bumanos destinados a la creación de una estructura de prevención organizada de acuerdo a las necesidades de cada área de negocio, así como un Servicio de Prevención mancomunado especializado por área de negocio; y a la redistribución de los técnicos especialistas de acuerdo a las nuevas necesidades; y se crea el Comité de Prevención, con la realización de reuniones entre la dirección corporativa y las áreas de negocio para el diseño, seguimiento y análisis de la gestión preventiva, y el desarrollo de grupos de trabajo específicos.

La comunicación es implantada mediante el Portal de Prevención de Riesgos Laborales para toda la organización, donde cada área de actividad tiene su propio sitio de colaboración de Prevención, y se difunde el compromiso con la gestión de entornos seguros y saludables, y la plantilla encuentra soporte eficaz de información, participación, consulta y gestión en materia preventiva; el Portal de Seguridad Vial con campañas temáticas y como foco de información y colaboración en la materia; la revista de FCC, con la sección fija "Bienestar" destinada a transmitir mensajes e informaciones relacionadas con la seguridad y salud de las personas en el ámbito laboral y personal; un buzón de Prevención a disposición del personal de FCC; y las campañas específicas para todos los grupos como: "Construimos puentes seguros, destinada a prevención de accidentes de tráfico", "Con mayor cuidado, destinada al cuidado y medidas preventivas para la seguridad de nuestros mayores", y "Educa tu mundo, juego PREVENLAND, concurso para los más pequeños en materia de seguridad y salud".

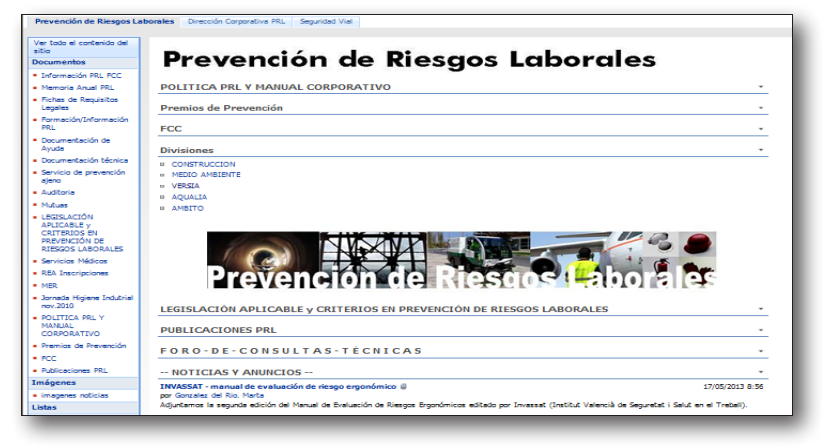



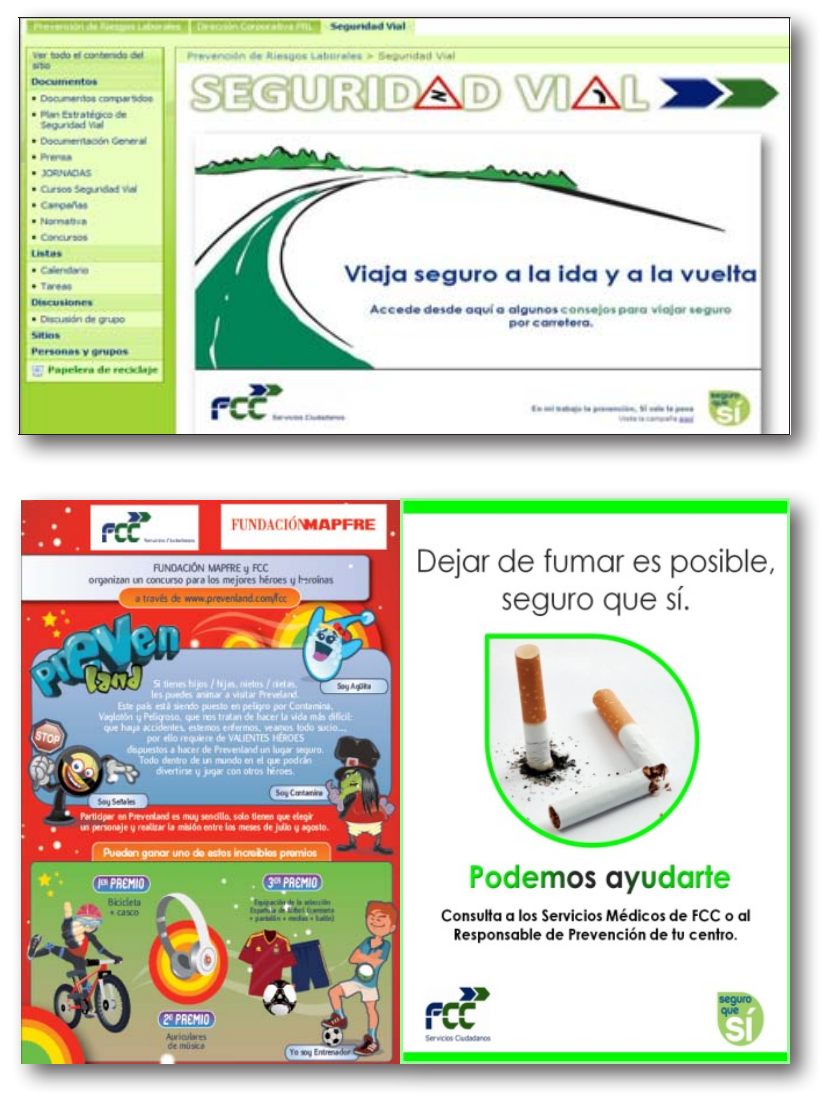

El estímulo juega un papel importante, algunas medidas implantadas son los Premios de Seguridad y Salud destinados a reconocer y premiar las mejores prácticas, intercambiar experiencias y asegurar que el conocimiento sea transmitido entre los distintos negocios; y el apoyo de la dirección con el vídeo del Director General de RRHH del Grupo a toda la organización.

\section{Implantación}

El objetivo es fomentar la cultura preventiva y favorecer la actitud positiva de los trabajadores hacia la prevención de riesgos laborales. El material gráfico es publicado en todos los centros, en todas las obras y centros no fijos, junto con vídeos de apoyo a la campaña.

El espíritu de la campaña es la asociación con el ámbito personal y familiar del trabajador; aportar un mensaje positivo de ánimo y felicitación por el trabajo bien hecho; fomentar el compañerismo y el trabajo en equipo; y la implicación de toda la organización.

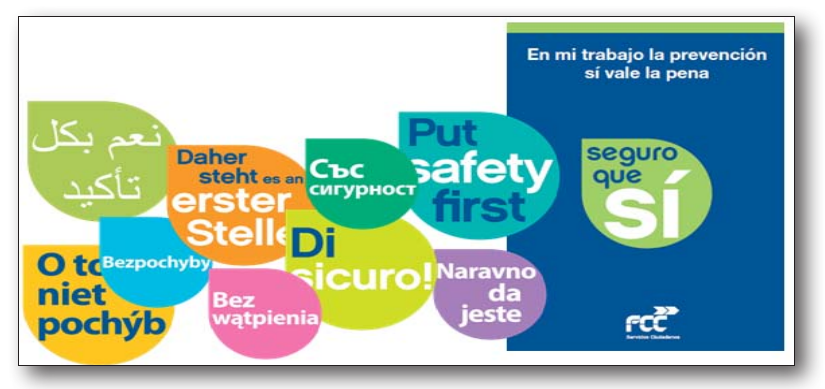




\section{Eficacia y resultados}

Constante reducción de la siniestralidad en los últimos ejercicios, dando como ejemplo una reducción del 14\% del índice de frecuencia y un 5\% el índice de gravedad en el último trienio.

Un sistema de prevención adecuado, con los recursos necesarios y la implicación de todos los participantes, desemboca en un claro aumento de la productividad de la empresa y una mayor eficiencia en el trabajo. Favoreciendo las relaciones entre los trabajadores y de éste con la propia empresa.

Implantación de sistemas dinámicos que fomentan la mejora continua, así como la participación y la generación de nuevas ideas y enfoques, asegurarán la constante capacidad de la empresa para adaptarse a las nuevas situaciones, provocando su eficacia y rentabilidad.

La ampliación del ámbito de estas acciones a todos los grupos de interés, y la sociedad en general, contribuye a la creación de una sociedad más humana, segura y responsable.

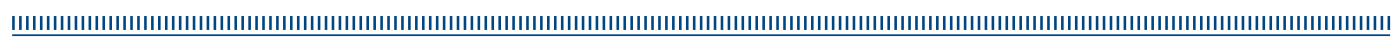

